\title{
Malaria vaccine: a new tool in the fight against
}

\section{malaria}

\section{Dr Edward Eremugo Kenyi}

\section{Editor-in-Chief, South Sudan Medical Journal}

In early October 2021 the World Health Organization (WHO) recommended the use of the first-ever malaria vaccine for children aged under 5 in sub-Saharan Africa and other regions with moderate to high $P$. falciparum malaria transmission - this is welcome news in South Sudan. ${ }^{[1]}$

The whole of South Sudan is endemic for malaria, with high transmission in the country throughout the year. Malaria is the leading cause of illness and death in children under five years. In 2019, the malaria incidence (all ages) was estimated at 246 per 1,000 populations (239 per 1,000 for children under-five-years) representing 5,067,464 cases. $^{[2]}$

The main methods deployed against malaria are malaria case management (early diagnosis and treatment), Insecticide Treated Nets (ITN), Indoor Residual Spray (IRS) and chemoprevention methods such as Intermittent Preventive Treatment of Malaria in Pregnant Women (IPTp) and Seasonal Malaria Chemoprevention (SMC) among others.

Despite the universal coverage in ITNs and usage of IRS in targeted areas of South Sudan, and the scale up of malaria diagnosis and treatment, current evidence shows that these core interventions have not had significant impact to interrupt malaria transmission. ${ }^{[2]}$ The 2017 Malaria Indicator Survey showed that only about $41.7 \%$ of children aged under five years had slept under an ITN the previous night, and that only $27 \%$ of households owned at least one ITN for every two people.

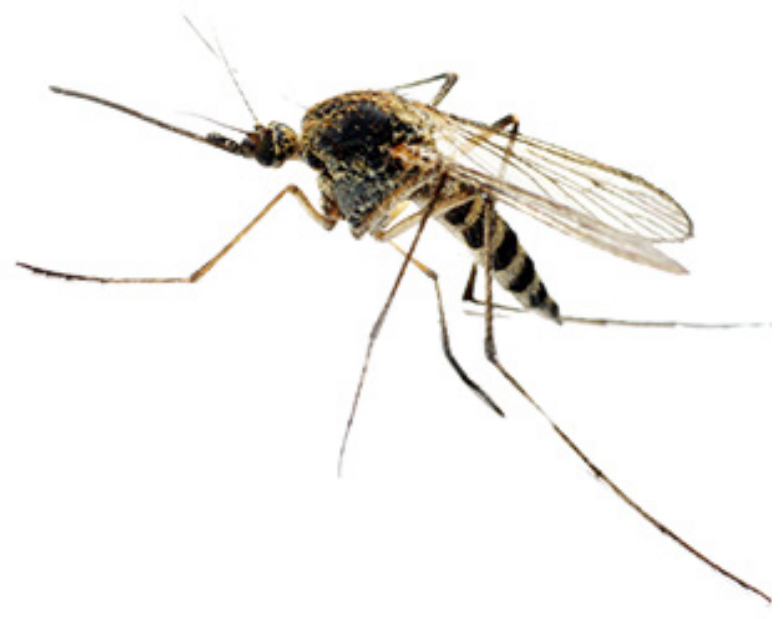

The malaria vaccine is an added tool in the arsenal deployed against malaria. When used in combination with the other prevention methods, the malaria vaccine can be highly effective. ${ }^{[3]}$

The vaccine, known by the acronym RTS,S, is the first malaria vaccine shown to provide partial protection against malaria in children under five, and is the firstever vaccine against a human parasite recommended for use by $\mathrm{WHO}$, following long-running clinical trials in Kenya, Ghana and Malawi. (See infographic on page 152) According to WHO, the vaccine should be provided in a schedule of 4 doses in children from 5 months of age in order to reduce malaria disease. ${ }^{[1]}$

For South Sudan, it is time to add the vaccine to the malaria strategy and plan a roll out and implementation guidelines. There is no time to waste.

References

1. World Health Organization, WHO recommends groundbreaking malaria vaccine for children at risk, News Release, 6 October 2021. https://bit. ly/3D4WrjB

Citation: Kenyi. Malaria vaccine: a new

2. Pasquale. Malaria Prevention Strategies in South Sudan. South Sudan Medical Journal 2020; 13(5):187-190. https://bit.ly/30qFamz

3. Chandramohan et al, Seasonal Malaria Vaccination with or without Seasonal Malaria Chemoprevention N Engl J Med 2021; 385:1005-1017 DOI: 10.1056/NEJMoa2026330

tool in the fight against malaria South Sudan Medical Journal 2021;14(4):114 (C) 2021 The Author (s) License: This is an open access article under CC BY-NC DOI: https://dx.doi.org/10.4314/ssmj. v14i4.1 